\title{
EXAMPLES OF SEMIRINGS OF ENDOMORPHISMS OF SEMIGROUPS
}

\author{
MIREILLE P. GRILLET
}

(Received 5 May 1969)

Communicated by G. B. Preston

Semirings of endomorphisms of semigroups form an important class of semirings. All examples which can be found in the literature concern semigroups with a subcommutative operation. We show that there exists a non-subcommutative semigroup whose endomorphisms form a semiring (this answers a question raised by Professor $\mathbf{A}$. $\mathrm{H}$. Clifford). We also give an example of a semigroup whose set of endomorphisms is not embeddable into a semiring; however it is the disjoint union of two semirings, but one of these semirings is not embeddable into a semiring with identity.

By a semiring, we understand a set together with two associative operations + and such that $x(y+z)=x y+x z$ and $(y+z) x=y x+x z$ hold identically. Let $R$ be a semiring; we denote by $(R,+)$ and $(R, \cdot)$ the additive and multiplicative semigroups of $R$. A zero of $R$ is an element 0 of $R$ which is together an additive identity and a multiplicative zero; an identity of $R$ is an identity of $(R,$.$) .$

An (additive) semigroup is said to be subcommutative if $x+y+z+t=$ $x+z+y+t$ holds identically. Semirings arise naturally as sets of endomorphisms of subcommutative semigroups with the induced addition as addition and the composition of mappings as multiplication.

In general the set of endomorphisms of any semigroup $S$ is a multiplicative semigroup, and is a semiring if and only if it is closed under addition (this is trivially equivalent to $f(x)+f(y)+g(x)+g(y)=f(x)+g(x)+f(y)+g(y)$ for all $x, y \in S, f, g \in \operatorname{End}(S))$. This is in general not satisfied. As an example of an extreme case, if $S$ is the bicyclic semigroup, then no sum $f+g$ is defined in $\operatorname{End}(S)$, except for $f=0$ or $g=0$ (where $0(x)=0$ for all $x \in S$ ).

Necessary and sufficient conditions on a semigroup $S$ under which End $(S)$ is a semiring are not known; an easy necessary condition is that $n x+n y=n(x+y)$ holds identically for all $n>1$, in which case the semigroup is said to be normal. This condition simply expresses that, if $I_{S}$ is the identity mapping of $S$, then $n I_{S}$ is an endomorphism of $S$ for all $n>1$. If $S$ is a normal semigroup, then, for any endomorphism $f$ of $S$ and all $n>1, n f$ is also an endomorphism of $S$. Many examples, in particular the example of section 2, show that the endomorphisms of a normal semigroup need not form a semiring. 


\section{A non-subcommutative semigroup whose endomorphisms form a semiring}

Let $S$ be the semigroup generated by a two elements set $X=\{a, b\}$, and subject to the relations $a=b+a$ and $2 a+2 b=2 a+b$. It is easy to check that $S$ is the set of all elements having one of the following forms: $n a, p b, r a+q b$ (where $n, p, q, r$ are positive integers, $q=1$ if $r \geqq 2$ ) with the addition given by the following table, where $p, r, p^{\prime}, r^{\prime} \geqq 1$ and $n, s, n^{\prime}, s^{\prime} \geqq 2$ :

\begin{tabular}{l|lllll}
\hline & $a$ & $n^{\prime} a$ & $p^{\prime} b$ & $a+r^{\prime} b$ & $s^{\prime} a+b$ \\
\hline$a$ & $2 a$ & $\left(n^{\prime}+1\right) a$ & $a+p^{\prime} b$ & $2 a+b$ & $\left(s^{\prime}+1\right) a+b$ \\
$n a$ & $(n+1) a$ & $\left(n+n^{\prime}\right) a$ & $n a+b$ & $(n+1) a+b$ & $\left(n+s^{\prime}\right) a+b$ \\
$p b$ & $a$ & $n^{\prime} a$ & $\left(p+p^{\prime}\right) b$ & $a+r^{\prime} b$ & $s^{\prime} a+b$ \\
$a+r b$ & $2 a$ & $\left(1+n^{\prime}\right) a$ & $a+\left(r+p^{\prime}\right) b$ & $2 a+b$ & $\left(s^{\prime}+1\right) a+b$ \\
$s a+b$ & $(s+1) a$ & $\left(s+n^{\prime}\right) a$ & $s a+b$ & $(s+1) a+b$ & $\left(s+s^{\prime}\right) a+b$ \\
\hline
\end{tabular}

Since $b+b+a+b=a+b \neq a+b+b=b+a+b+b$, we see that $S$ is not a subcommutative semigroup. We want to show that End $(S)$ still forms a semiring. For any $u, v \in S$ satisfying $u=u+v$ and $2 u+2 v=2 u+v$, there exists an endomorphism $f$ of $S$ such that $f(a)=u$ and $f(b)=v$. Looking at the multiplication table of $S$, we obtain:

Proposition 1. Any endomorphism of $S$ is determined by:

$$
f(a)=n a+q b ; f(b)=p b \text { for some } n, p>0, q \geqq 0 ;
$$

then, for all $i a+j b \in S$ with $i \geqq 0, j \geqq 0$,

$$
f(i a+j b)=n i a+(\alpha(i) q+j p) b,
$$

where $\alpha(i)=1$ if $i \neq 0$ and $\alpha(i)=0$ if $i=0$.

Proof. It is clear on the definition of the addition in $S$ that $f(a)=f(b)+f(a)$ is equivalent to (1). Also, if $f(a)$ and $f(b)$ are given by (1), we have:

$$
2 f(a)+2 f(b)=n a+q b+n a+q b+2 p b=n a+q b+n a+q b+p b=2 f(a)+f(b)
$$

so that the mapping $f$ of $S$ to $S$ defined by

$$
f(i a+j b)=i f(a)+j f(b)=i(n a+q b)+j p b=n i a+(\alpha(i) q+j p) b
$$

is indeed an endomorphism of $S$.

TheOrem 2. End $(S)$ is a semiring.

PROOF. To show that $\operatorname{End}(S)$ is closed under addition, let $f, g \in \operatorname{End}(S)$ and write $f(a)=n a+q b, f(b)=p b, g(a)=r a+s b, g(b)=t b$ for some positive in- 
tegers $n, p, r, t$ and non negative integers $q, s$. We then have to prove that, for any two elements $x=i a+j b, y=i^{\prime} a+j^{\prime} b$ of $S$, the two expressions

$$
A=f(x)+f(y)+g(x)+g(y) \text { and } B=f(x)+g(x)+f(y)+g(y)
$$

are equal.

i) First, if $i^{\prime} \neq 0$, then $r i^{\prime} \neq 0$ and

$$
\begin{aligned}
A & =f(i a+j b)+f\left(i^{\prime} a+j^{\prime} b\right)+g(i a+j b)+g\left(i^{\prime} a+j^{\prime} b\right) \\
& =n i a+(\alpha(i) q+j p) b+n i^{\prime} a+\left(q+p j^{\prime}\right)+r i a+(\alpha(i) s+t j) b+r i^{\prime} a+\left(s+t j^{\prime}\right) b \\
& =(n+r)\left(i+i^{\prime}\right) a+\left(s+t j^{\prime}\right) b ;
\end{aligned}
$$

similarly $B=(n+r)\left(i+i^{\prime}\right) a+\left(s+t j^{\prime}\right) b$ so that $A=B$.

ii) If now $i^{\prime}=0$, but $i \neq 0$, then the above expression of $A$ simplifies to:

$$
\begin{aligned}
& A=n i a+(q+p j) b+p j^{\prime} b+r i a+(s+t j) b+t j^{\prime} b, \\
& B=n i a+(q+p j) b+r i a+(s+t j) b+p j^{\prime} b+t j^{\prime} b .
\end{aligned}
$$

Since $r i \neq 0$, we obtain $A=(n+r) a+\left(s+t j+t j^{\prime}\right) b$ and $B=(n+r) a+(s+t j+$ $\left.p j^{\prime}+t j^{\prime}\right) b$. If $j^{\prime}=0$, then trivially $A=B$. If $j^{\prime} \neq 0$, then $s+t j+p j^{\prime}+t j^{\prime} \geqq 1$ and $s+t j+t j^{\prime} \geqq 1 ;$ also $(n+r) i \geqq 2$, whence $A=B$.

(iii) Finally, when $i=i^{\prime}=0, A=\left(j+j^{\prime}\right)(p+t) b=B$. Thus End (S) is closed under addition whence is a semiring.

There exists a bijective correspondance between endomorphisms of $S$ and the set of triples $(n, q, p)$ such that $f(a)=n a+q b, f(b)=p b$, which permits us to represent the semiring $R$ as a semiring of triples. More precisely, if $r \geqq 0$ and $s \geqq 0$, let $\beta_{s}(r)=r$ if $r=0$ or $s \leqq 1$ and $\beta_{s}(r)=1$ otherwise; then we have the following:

Corollary 3 . The set of all triples $(n, q, p)$ (where $n>0, q \geqq 0, p>0$ are such that $q \leqq 1$ if $n>1$ ) together with the following operations

$$
\begin{aligned}
(n, q, p)+\left(n^{\prime}, q^{\prime}, p^{\prime}\right) & =\left(n+n^{\prime}, q^{\prime}, p+p^{\prime}\right), \\
(n, q, p)\left(n^{\prime}, q^{\prime}, p^{\prime}\right) & =\left(n n^{\prime}, \beta_{n n},\left(q+p q^{\prime}\right), p p^{\prime}\right)
\end{aligned}
$$

is a semiring isomorphis to $\operatorname{End}(S)$.

Proof. It follows immediately from the definition of the operations in $\operatorname{End}(S)$ that, if $f(a)=n a+q b, f(b)=p b, g(a)=n^{\prime} a+q^{\prime} b$ and $g(b)=p^{\prime} b$ for some $f, g \in \operatorname{End}(S)$, then $(f+g)(a)=\left(n+n^{\prime}\right) a+q^{\prime} b, \quad(f+g)(b)=\left(p+p^{\prime}\right) b$; $(f g)(a)=n n^{\prime} a+\left(q+p q^{\prime}\right) b=n n^{\prime} a+\beta_{n n^{\prime}}\left(q+p q^{\prime}\right) b,(f g)(b)=p p^{\prime} b$. Thus the result holds. 


\section{A semigroup whose set of endomorphisms is not embeddable into a semiring}

Let $S^{0}$ be the semigroup obtained by adjunction of a formal identity of $S$. For any endomorphism $f$ of $S^{0}, f(0)=0$ holds, since 0 is the unique idempotent of $S^{0}$; moreover observe that, if $f(a)=0$, then $0=f(a)=f(a)+f(b)=0+f(b)$ $=f(b)$ implies $f(b)=0$; hence the only endomorphism of $S^{0}$ which sends a upon 0 is the trivial endomorphism 0 defined by: $0(x)=0$ for all $x \in S^{0}$. Therefore any endomorphism of $S^{0}$ must be of one of the following types:

(A): $f(a), f(b) \in S$, so that $f$ is the trivial extension to $S^{0}$ of an endomorphism of $S$; we shall identify the set of such endomorphisms with End $(S)$ in the obvious way and therefore consider $\operatorname{End}(S)$ as a subset of $\operatorname{End}\left(S^{0}\right)$.

(B): $f(a) \in S^{0}, f(b)=0$; observe that for any $u=n a+p b \in S^{0}, 2 u+20=$ $2 u+0$ and $u=0+u$, whence there exists an endomorphism of type (B), say $f$, such that $f(a)=u, f(b)=0$. Clearly, if $f(a)=n a+p b$, for all $i a+j b \in S^{0}(i, j \geqq 0)$,

$$
f(i a+j b)=i f(a)=i(n a+p b)=n i a+\delta_{n}(i) p b,
$$

where $\delta_{n}(0)=0, \delta_{0}(i)=i$ and $\delta_{n}(i)=1$ if in $\neq 0$. We denote by $R$ the set of such endomorphisms of $S^{0}$. Clearly $\operatorname{End}\left(S^{0}\right)=R \cup \operatorname{End}(S), R \cap \operatorname{End}(S)=\emptyset$.

THEOREM 4. With the induced addition as addition and the composition of mappings as multiplication, $R$ is a semiring with zero.

Proor. Again we need to show that $A=f(x)+f(y)+g(x)+g(y)$ and $B=$ $f(x)+g(x)+f(y)+g(y)$ are equal for all $f, g \in R$ and $x, y \in S^{0}$. This is trivial in case when $f=0$ or $g=0$, or in case $x(y)$ equals $j b(j \geqq 0)$. Therefore let $x=i a+$ $j b, y=i^{\prime} a+j^{\prime} b$ where $i, i^{\prime}>0, j, j^{\prime} \geqq 0$; let $f$ and $g$ be defined by $f(a)=n a+p b$, $g(a)=r a+t b(n, p, r, t \geqq 0), f(b)=g(b)=0$, with $n$ and $p(r$ and $t)$ not both zero.

i) Assume first that $r \neq 0$; then $\delta_{r}(i)=\delta_{r}\left(i^{\prime}\right)=1$ whence

$$
A=n i a+\delta_{n}(i) p b+n i^{\prime} a+\delta_{n}\left(i^{\prime}\right) p b+r i a+t b+r i^{\prime} a+t b=(n+r)\left(i+i^{\prime}\right) a+t b ;
$$

similarly $B=(n+r)\left(i+i^{\prime}\right) a+t b$; thus $A=B$.

ii) If $r=0, n \neq 0$, then $\delta_{r}(i)=i, \delta_{r}\left(i^{\prime}\right)=i^{\prime}, \delta_{n}(i)=\delta_{n}\left(i^{\prime}\right)=1$; also $t \neq 0$ since $r a+t b \in S$. Then

$$
\begin{aligned}
& A=n i a+p b+n i^{\prime} a+p b+t i b+t i^{\prime} b=n\left(i+i^{\prime}\right) a+\left(p+t\left(i+i^{\prime}\right)\right) b ; \\
& B=n i a+p b+t i b+n i^{\prime} a+p b+t i^{\prime} b=b\left(i+i^{\prime}\right) a+\left(p+t i^{\prime}\right) b .
\end{aligned}
$$

Also $n\left(i+i^{\prime}\right) \geqq 2, p+t\left(i+i^{\prime}\right) \geqq 1, p+t i^{\prime} \geqq 1$; whence $A=B$.

(iii) Finally, in case $r=n=0$, then $A=(p+t)\left(i+i^{\prime}\right) b=B$ trivially.

As any element of $R$ is determined by its action on $a$, it is possible to represent $R$ by means of ordered pairs as follows: 
CoRollary 5 . The semiring $R$ is isomorphic to the semiring consisting of all pairs $(n, p)$ of non negative integers $n, p$ such that $p \geqq 1$ whenever $n \geqq 2$ with the following operations:

$$
\begin{aligned}
(n, p)+\left(n^{\prime}, p^{\prime}\right) & =\left(n+n^{\prime}, \beta_{n+n^{\prime}}\left(\rho\left(n^{\prime}\right) p+p^{\prime}\right)\right) \\
(n, p)\left(n^{\prime}, p^{\prime}\right) & =\left(n n^{\prime}, \beta_{n n^{\prime}}\left(\delta_{n}\left(n^{\prime}\right) P\right)\right),
\end{aligned}
$$

where $\beta$ and $\delta$ are as in Corollary 3 and formula (3) and $\rho\left(n^{\prime}\right)=0$ if $n^{\prime} \neq 0$, $\rho\left(n^{\prime}\right)=0$ if $n^{\prime} \neq 0$.

REMARK. Clearly $R$ has no identity and its addition is not subcommutative; this will also result from the following:

Proposition 6. $R$ is not embeddable into a semiring with identity.

Proof. If $R$ were embeddable into a semiring $R^{\prime}$ with identity 1 , then, for all $\dot{x}, y, z \in R$, the relation $y+x y+z+x z=y+z+x y+x z$ would hold in $R$, since both members of this relations are equal to $(1+x)(y+z)$ in $R^{\prime}$. But, if $x=(1,0)$ $=y$ and $z=(0,1)$, then

$$
y+x y+z+x z=(2,1) \neq(2,0)=y+z+x y+x z .
$$

Thus $R$ is not embeddable into a semiring with identity.

COROLl ARY 7. End $\left(S^{0}\right)$ is not embeddable into a semiring.

Proof. If $\operatorname{End}\left(S^{0}\right)$ were embeddable into a semiring $R^{\prime}$, then $R$ would be embeddable into a semiring with identity: $\left\{r \in R^{\prime} ; r I_{S^{0}}=I_{S^{\circ}} r=r\right\}$ (where $I_{S^{\circ}}$ is the identity mapping of $S^{0}$ ) which is clearly a subsemiring of $R^{\prime}$ containing $\operatorname{End}\left(S^{0}\right)$ and has $I_{S^{0}}$ as identity. This is impossible by propostion 6 .

Louisiana State University in New Orleans 